\title{
SYMMETRIC SEMI-CLASSICAL ORTHOGONAL POLYNOMIALS OF CLASS ONE ON $q$-QUADRATIC LATTICES
}

\author{
G. FILIPUK AND M.N. REBOCHO
}

\begin{abstract}
In this paper we study discrete semi-classical orthogonal polynomials on non-uniform lattices. In the symmetric class one case we give a closed form expression for the recurrence coefficients of orthogonal polynomials.
\end{abstract}

KeYwords: Orthogonal polynomials; Divided-difference operator; Non-uniform lattices; semi-classical class.

Math. Subject Classification (2000): 33C45, 33C47, $42 \mathrm{C} 05$.

\section{Motivation}

Orthogonal polynomials on $q$-quadratic lattices are part of the discrete families of orthogonal polynomials. These families are widely spread in the literature of special functions and applications. Some works we refer to the interested reader include $[7,13,14]$, where a comprehensive approach to orthogonal polynomials is given, gathering, amongst many other topics, the analysis of divided difference operators, related problems on classification, connections with the Sturm-Louville theory, etc.

In the present paper we consider the general divided-difference operator $\mathbb{D}$ [10, Eq. (1.1)] having the basic property of leaving a polynomial of degree $n-1$ when applied to a polynomial of degree $n$. It is well-know that $\mathbb{D}$ yields the Askey-Wilson operator [1] under appropriate specifications. The related lattice, commonly known as a non-uniform lattice, is obtained from a conic defined by (2) [11, Sec. 2] and involves the $q$-quadratic lattice due to its parametric representations (more details are given in Section 2.1). The main problem to be analysed in the present paper lies within the so-called direct problem (see, e.g., [17]): to extract information on recurrence coefficients of orthogonal polynomials, given some data on the corresponding Stieltjes

Received September 16, 2018.

G. Filipuk acknowledges the support of Alexander von Humboldt Foundation.

The work of M.N. Rebocho is partially supported by the Centre for Mathematics of the University of Coimbra - UID/MAT/00324/2013, funded by the Portuguese Government through FCT/MCTES and co-funded by the European Regional Development Fund through the Partnership Agreement PT2020. 
function or the orthogonality measure. We take the difference equation satisfied by the Stieltjes functions, say $A \mathbb{D} S=C \mathbb{M} S+D$, where $A, C, D$ are polynomials, subject to restrictions $\operatorname{deg}(A) \leq 3, \operatorname{deg}(C) \leq 2$. According to the classification from [8], this is the so-called class one (see Section 2.2). Our main goal is to give a closed form expression for the recurrence coefficients of orthogonal polynomials in the symmetric case, that is, when one of the recurrence coefficients is zero (cf. Section 3). Such a closed formula is given in terms of the lattice as well as in terms of the polynomials $A, C, D$. To the best of authors' knowledge, these results are new in the literature. Furthermore, as the calculus on non-uniform lattices generalizes the calculus on lattices of lower complexity (see [13], [18, Sec. 2]), our results may be regarded as a generalization of some of the results on semi-classical orthogonal polynomials of class one, for instance: [12], on symmetric orthogonal polynomials when $\mathbb{D}$ is Hahn's difference operator; [2], on orthogonal polynomials when $\mathbb{D}$ is the derivative operator; [4], on orthogonal polynomials when $\mathbb{D}$ is the forward difference operator.

The paper is organized as follows. In Section 2 we give the definitions and state the basic results which will be used in the forthcoming sections. In Section 3 we present the main results of the paper, namely, a closed form expression for the recurrence coefficients of the symmetric orthogonal polynomials (see Theorem 1).

\section{Preliminary Results}

2.1. The General Divided Difference Operator, q-Quadratic Lattices, and Orthogonal Polynomials. We consider the divided difference operator $\mathbb{D}$ given in $[10$, Eq.(1.1)], with the property that $\mathbb{D}$ leaves a polynomial of degree $n-1$ when applied to a polynomial of degree $n$. The operator $\mathbb{D}$, defined on the space of arbitrary functions, is given in terms of two functions, $y_{1}, y_{2}$,

$$
(\mathbb{D} f)(x)=\frac{f\left(y_{2}(x)\right)-f\left(y_{1}(x)\right)}{y_{2}(x)-y_{1}(x)} .
$$

The functions $y_{1}, y_{2}$ may be defined as the two $y$-roots of the quadratic equation

$$
\hat{a} y^{2}+2 \hat{b} x y+\hat{c} x^{2}+2 \hat{d} y+2 \hat{e} x+\hat{f}=0, \quad \hat{a} \neq 0 .
$$

In the present paper we shall consider the $q$-quadratic case, related to the socalled non-uniform lattices, which appears whenever $\lambda \tau \neq 0, \lambda=\hat{b}^{2}-\hat{a} \hat{c}, \tau=$ 
$\left(\left(\hat{b}^{2}-\hat{a} \hat{c}\right)\left(\hat{d}^{2}-\hat{a} \hat{f}\right)-(\hat{b} \hat{d}-\hat{a} \hat{e})^{2}\right) / \hat{a}$. In such a case, $y_{1}, y_{2}$ are given by

$$
y_{1}(x)=p(x)-\sqrt{r(x)}, \quad y_{2}(x)=p(x)+\sqrt{r(x)}
$$

with $p, r$ polynomials given by

$$
p(x)=-\frac{\hat{b} x+\hat{d}}{\hat{a}}, \quad r(x)=\frac{\lambda}{\hat{a}^{2}}\left(x+\frac{\hat{b} \hat{d}-\hat{a} \hat{e}}{\lambda}\right)^{2}+\frac{\tau}{\hat{a} \lambda} .
$$

We shall use the notation $\Delta_{y}=y_{2}-y_{1}$. From (3), it follows that

$$
\Delta_{y}=2 \sqrt{r} \text {. }
$$

For the $q$-quadratic case there is a parametric representation of the conic (2), $x=x(s), y=y(s)$, such that [11, pp. 254-255]

$$
x(s)=x_{c}+\xi \sqrt{\hat{a}}\left(q^{s}+q^{-s}\right), \quad y(s)=y_{c}+\xi \sqrt{\hat{c}}\left(q^{s-1 / 2}+q^{-s+1 / 2}\right),
$$

$x_{c}=(\hat{a} \hat{e}-\hat{b} \hat{d}) / \lambda, y_{c}=(\hat{c} \hat{d}-\hat{b} \hat{e}) / \lambda, \xi^{2}=\tilde{f} /(4 \lambda), \tilde{f}=\hat{f}-\hat{a} y_{c}^{2}-2 \hat{b} x_{c} y_{c}-\hat{c} x_{c}^{2}$, and $q$ defined by

$$
q+q^{-1}=\frac{4 \hat{b}^{2}}{\hat{a} \hat{c}}-2 .
$$

We have $y_{1}(x(s))=y(s), y_{2}(x(s))=y(s+1)$. Thus, in the account of $(3)$, we have

$$
p(x(s))-\sqrt{r(x(s))}=y(s), \quad p(x(s))+\sqrt{r(x(s))}=y(s+1)
$$

and

$$
y(s+1)+y(s)=2 p(x(s)), \quad(y(s+1)-y(s))^{2}=4 r(x(s)) .
$$

In the present paper we shall operate with $\mathbb{D}$ given in its general form (1). We now define other operators related to (1). Firstly, by defining $\mathbb{E}_{1}$ and $\mathbb{E}_{2}$ (see [10]), acting on arbitrary functions $f$ as

$$
\left(\mathbb{E}_{1} f\right)(x)=f\left(y_{1}(x)\right), \quad\left(\mathbb{E}_{2} f\right)(x)=f\left(y_{2}(x)\right),
$$

the so-called companion operator of $\mathbb{D}$ is defined as (see [10])

$$
(\mathbb{M} f)(x)=\frac{\left(\mathbb{E}_{1} f\right)(x)+\left(\mathbb{E}_{2} f\right)(x)}{2} .
$$

Note that $\mathbb{M} f$ is a polynomial whenever $f$ is a polynomial. Furthermore, if $\operatorname{deg}(f)=n$, then $\operatorname{deg}(\mathbb{M} f)=n$. Indeed, in the account of $(3)$, one has

$$
\mathbb{E}_{j}\left(x^{n}\right)=\left(p(x)+(-1)^{j} \sqrt{r(x)}\right)^{n}, \quad j=1,2 .
$$


From the binomial identity

$$
(p \pm \sqrt{r})^{n}=\sum_{i=0}^{n}\left(\begin{array}{c}
n \\
i
\end{array}\right) p^{i}( \pm \sqrt{r})^{n-i}
$$

we get, for $n$ odd,

$\mathbb{D} x^{n}=\sum_{i=0}^{(n-1) / 2}\left(\begin{array}{c}n \\ 2 i\end{array}\right) p^{2 i} r^{(n-2 i-1) / 2}, \quad \mathbb{M} x^{n}=\sum_{i=0}^{(n-1) / 2}\left(\begin{array}{c}n \\ 2 i+1\end{array}\right) p^{2 i+1} r^{(n-2 i-1) / 2}$,

and for $n$ even,

$$
\mathbb{D} x^{n}=\sum_{i=0}^{(n-2) / 2}\left(\begin{array}{c}
n \\
2 i+1
\end{array}\right) p^{2 i+1} r^{(n-2 i-2) / 2}, \quad \mathbb{M} x^{n}=\sum_{i=0}^{n / 2}\left(\begin{array}{c}
n \\
2 i
\end{array}\right) p^{2 i} r^{(n-2 i) / 2} .
$$

In the remainder of the paper we use the following notation:

$$
\mathbb{D} x^{n}=\sum_{k=0}^{n-1} d_{n, k} x^{k}, \quad \mathbb{M} x^{n}=\sum_{k=0}^{n} m_{n, k} x^{k} .
$$

We shall consider orthogonal polynomials related to a (formal) Stieltjes function defined by

$$
S(x)=\sum_{n=0}^{+\infty} u_{n} x^{-n-1}
$$

where $\left(u_{n}\right)$, the sequence of moments, is such that $\operatorname{det}\left[u_{i+j}\right]_{i, j=0}^{n} \neq 0, n \geq 0$, and, without loss of generality, $u_{0}=1$. The orthogonal polynomials related to $S, P_{n}, n \geq 0$, are taken to be monic, and we will denote the sequence $\left\{P_{n}\right\}_{n \geq 0}$ by SMOP.

Monic orthogonal polynomials satisfy a three-term recurrence relation [16]

$$
P_{n+1}(x)=\left(x-\beta_{n}\right) P_{n}(x)-\gamma_{n} P_{n-1}(x), \quad n=0,1,2, \ldots,
$$

with $P_{-1}(x)=0, P_{0}(x)=1$, and $\gamma_{n} \neq 0, n \geq 1, \gamma_{0}=1$.

The quantities $\beta_{n}, \gamma_{n}$ are called the recurrence coefficients of $\left\{P_{n}\right\}_{n \geq 0}$.

Another important sequence, related to $\left\{P_{n}\right\}_{n \geq 0}$, is the sequence of associated polynomials of the first kind, denoted by $\left\{P_{n}^{(1)}\right\}_{n \geq 0}$, which satisfies the three term recurrence relation

$$
P_{n}^{(1)}(x)=\left(x-\beta_{n}\right) P_{n-1}^{(1)}(x)-\gamma_{n} P_{n-2}^{(1)}(x), \quad n=1,2, \ldots
$$


with $P_{-1}^{(1)}(x)=0, P_{0}^{(1)}(x)=1$.

In the framework of the Hermite-Padé Approximation (see [9]), the polynomials $P_{n}$ are the diagonal Padé denominators of (12), and the polynomials $P_{n-1}^{(1)}$ are the numerator polynomials, thus, also determined by the relation

$$
S(x)-P_{n-1}^{(1)}(x) / P_{n}(x)=\mathcal{O}\left(x^{-2 n-1}\right), \quad x \rightarrow \infty .
$$

\subsection{Semi-Classical Orthogonal Polynomials on Non-Uniform Lat-} tices: the Class One and General Difference Equations. Semi-classical orthogonal polynomials on non-uniform lattices may be defined by a difference equation for the Stieltjes function $[10,18]$,

$$
A \mathbb{D} S=C \mathbb{M} S+D
$$

where $A, C, D$ are irreducible polynomials (in $x$ ). In general, the polynomials $A, C, D$ in (14) satisfy, in the account of (1), (8), and (12),

$$
\operatorname{deg}(A) \leq m+2, \operatorname{deg}(C) \leq m+1, \operatorname{deg}(D) \leq m,
$$

where $m$ is some nonnegative integer. When $m=0$ we get the so-called classical polynomials $[6,15]$.

In the present paper we will study class one (see [8, Def. 8 and Th. 9]), that is, we will take the difference equation (14) for $S$ with $m=1$ in (15), under the following condition (in order to avoid degenerate cases):

$$
\operatorname{deg}(A)=3,1 \leq \operatorname{deg}(C) \leq 2 \text { or } \operatorname{deg}(A)<3, \operatorname{deg}(C)=2 .
$$

A very useful result on semi-classical orthogonal polynomials concerns the difference equations studied recently in $[3,5,11]$. In particular, SMOP related to (14) satisfy the following difference equations, for all $n \geq 0$ :

$$
\begin{aligned}
& A_{n+1} \mathbb{D} P_{n+1}=\left(l_{n}-C / 2\right) \mathbb{M} P_{n+1}+\Theta_{n} \mathbb{M} P_{n}, \\
& A_{n+1} \mathbb{D} P_{n}^{(1)}=\left(l_{n}+C / 2\right) \mathbb{M} P_{n}^{(1)}+D \mathbb{M} P_{n+1}+\Theta_{n} \mathbb{M} P_{n-1}^{(1)},
\end{aligned}
$$

with

$$
A_{n+1}=A+\frac{\Delta_{y}^{2}}{2} \pi_{n}
$$


Here $\Delta_{y}$ is defined in (5) and $l_{n}, \Theta_{n}, \pi_{n}$ are polynomials in $x$ satisfying, for all $n \geq 0$,

$$
\begin{aligned}
& \pi_{n+1}=-\frac{1}{2} \sum_{k=0}^{n+1} \frac{\Theta_{k-1}}{\gamma_{k}} \\
& l_{n+1}+l_{n}+\mathbb{M}\left(x-\beta_{n+1}\right) \frac{\Theta_{n}}{\gamma_{n+1}}=0 \\
& -A+\mathbb{M}\left(x-\beta_{n+1}\right)\left(l_{n+1}-l_{n}\right)-\frac{\Delta_{y}^{2}}{2}\left(\pi_{n+1}+\pi_{n}\right)+\Theta_{n+1}=\frac{\gamma_{n+1}}{\gamma_{n}} \Theta_{n-1},
\end{aligned}
$$

with initial conditions

$$
\begin{aligned}
& \pi_{-1}=0, \pi_{0}=-D / 2, \\
& \Theta_{-1}=D, \Theta_{0}=A-\frac{\Delta_{y}^{2}}{4} D-\left(l_{0}-C / 2\right) \mathbb{M}\left(x-\beta_{0}\right), \\
& l_{-1}=C / 2, l_{0}=-\mathbb{M}\left(x-\beta_{0}\right) D-C / 2 .
\end{aligned}
$$

\section{Main Results: the Symmetric Orthogonal Polynomi- als of Class One}

The symmetric families of class one satisfy (13) with $\beta_{n}=0, n \geq 0$, that is,

$$
P_{n+1}(x)=x P_{n}(x)-\gamma_{n} P_{n-1}(x), \quad n=0,1,2, \ldots,
$$

and they are related to the Stieltjes functions such that (16) holds. Let us now proceed to the determination of the recurrence coefficients $\gamma_{n}$.

The polynomials $A_{n}, l_{n}, \Theta_{n}, \pi_{n}$ in the difference equations from the previous section satisfy $\operatorname{deg}\left(A_{n}\right)=3, \operatorname{deg}\left(l_{n}\right)=2, \operatorname{deg}\left(\Theta_{n}\right)=\operatorname{deg}\left(\pi_{n}\right)=1$ [3] We set

$$
\begin{aligned}
& A(x)=a_{3} x^{3}+a_{2} x^{2}+a_{1} x+a_{0}, C(x)=c_{2} x^{2}+c_{1} x+c_{0}, \\
& D(x)=d_{1} x+d_{0}, A_{n}(x)=a_{n, 3} x^{3}+a_{n, 2} x^{2}+a_{n, 1} x+a_{n, 0}, \\
& l_{n}(x)=\ell_{n, 2} x^{2}+\ell_{n, 1} x+\ell_{n, 0}, \Theta_{n}(x)=\Theta_{n, 1} x+\Theta_{n, 0}, \pi_{n}=\pi_{n, 1} x+\pi_{n, 0}, \\
& p(x)=p_{1} x+p_{0}, r(x)=r_{2} x^{2}+r_{1} x+r_{0} .
\end{aligned}
$$

In accordance with (16), we will take the case

$$
\left|a_{3}\right|+\left|c_{2}\right| \neq 0 .
$$

In the following we shall also assume $p_{1} \neq 0, p_{1}^{2}-r_{2} \neq 0$, that is, $\hat{a} \hat{b} \hat{c} \neq 0$ in (2). 
The polynomial $D$ is defined in terms of the polynomials $A, C$. Indeed, by collecting the coefficients in (24) and (25), we get

$$
d_{1}=\frac{-\left(a_{3}+c_{2} p_{1}\right)}{p_{1}^{2}-r_{2}}, \quad d_{0}=\frac{-a_{2}-c_{1} p_{1}-c_{2} p_{0}+\left(r_{1}-2 p_{0} p_{1}\right) d_{1}}{p_{1}^{2}-r_{2}} .
$$

Theorem 1. Let $\left\{P_{n}\right\}_{n \geq 0}$ be a SMOP related to a Stieltjes function $S$ satisfying $A(x) \mathbb{D} S(x)=C(x) \mathbb{M} S(x)+D(x)$ with $\operatorname{deg}(A) \leq 3, \operatorname{deg}(C) \leq 2$, $\operatorname{deg}(D) \leq 1$ under condition (26) in the previous notation. Let the recurrence relation

$$
P_{n+1}(x)=x P_{n}(x)-\gamma_{n} P_{n-1}(x), \quad n=0,1,2, \ldots
$$

hold with $\gamma_{0}=1$. Under the previous notations we have, for all $n \geq 0$,

$$
\begin{aligned}
\gamma_{n+2} & =\frac{\gamma_{1} T_{0} D\left(x_{0}\right)+\sum_{k=0}^{n} \zeta_{k} T_{k}}{T_{n} T_{n+1}}, \\
\gamma_{1} & =\frac{\left(a_{1}+c_{1} p_{0}+c_{0} p_{1}+d_{0}\left(2 p_{0} p_{1}-r_{1}\right)+d_{1}\left(p_{0}^{2}-r_{0}\right)\right)\left(p_{1}^{2}-r_{2}\right)}{-a_{3}+c_{2} p_{1}+2 d_{1}\left(r_{2}+p_{1}^{2}\right)},
\end{aligned}
$$

where

$$
x_{0}=-p_{0} / p_{1}, \zeta_{n}=A\left(x_{0}\right)+2 r\left(x_{0}\right)\left(\pi_{n+1}+\pi_{n}\right)\left(x_{0}\right), T_{n}=\Theta_{n}\left(x_{0}\right) / \gamma_{n+1} .
$$

The quantities $\zeta_{n}, T_{n}$ are given in terms of the polynomials $A, C$ as well as of $p, r$ defined in (4). Indeed, there holds the following explicit formulae. For all $n \geq 0$,

$$
\begin{aligned}
& \frac{\Theta_{n+1,1}}{\gamma_{n+2}}=\frac{q^{-n-1}(q+1)\left(q^{2 n+2}-1\right)}{(q-1) p_{1}} \ell_{0,2}+\frac{q^{-n-1}\left(q^{2 n+3}-1\right)}{q-1} \frac{\Theta_{0,1}}{\gamma_{1}}, \\
& \pi_{n+1,1}=-\frac{d_{1}}{2}-\frac{\Theta_{0,1}}{2 \gamma_{1}}-\left(1-q^{-n}\right)\left(\frac{(q+1)\left(q^{n+1}-1\right)}{2 p_{1}(q-1)^{2}} \ell_{0,2}-\frac{\left(q^{n+2}-1\right)}{2(q-1)^{2}} \frac{\Theta_{0,1}}{\gamma_{1}}\right),
\end{aligned}
$$

$$
\frac{\Theta_{n+1,0}}{\gamma_{n+2}}=2\left(\pi_{n+1,0}-\pi_{n+2,0}\right),
$$

$\pi_{n+1,0}=\prod_{k=0}^{n} s_{k}\left(\pi_{0,0}+\sum_{k=0}^{n}\left(\prod_{j=0}^{k} s_{j}\right)^{-1} t_{k}\right)$, 
with

$$
\begin{aligned}
s_{n} & =\frac{f_{n, 0}+2 p_{1}}{2 p_{1}-f_{n+1,0}} \\
t_{n} & =\frac{f_{n+1}+f_{n}+f_{n+1,1} \pi_{n+1,1}+f_{n, 1} \pi_{n, 1}+p_{0} \Theta_{n, 1} / \gamma_{n+1}}{2 p_{1}-f_{n+1,0}} \\
f_{n, 0} & =\frac{2 r_{2} d_{n+1, n}}{m_{n+1, n+1}} \\
f_{n, 1} & =\frac{1}{m_{n+1, n+1}}\left(2 r_{2}\left(d_{n+1, n-1}-d_{n+1, n} \frac{m_{n+1, n}}{m_{n+1, n+1}}\right)+2 r_{1} d_{n+1, n}\right) \\
f_{n} & =\frac{c_{1}}{2}+\frac{1}{m_{n+1, n+1}}\left(a_{3}\left(d_{n+1, n-1}-d_{n+1, n} \frac{m_{n+1, n}}{m_{n+1, n+1}}\right)+a_{2} d_{n+1, n}\right)
\end{aligned}
$$

and the initial conditions

$$
\begin{gathered}
\ell_{0,2}=-p_{1} d_{1}-\frac{c_{2}}{2}, \frac{\Theta_{0,1}}{\gamma_{1}}=\frac{-a_{3}+c_{2} p_{1}+2 d_{1}\left(p_{1}^{2}+r_{2}\right)}{p_{1}^{2}-r_{2}}, \pi_{0,1}=-\frac{d_{1}}{2}, \quad \text { (39) } \\
\pi_{0,0}=-\frac{d_{0}}{2}, \frac{\Theta_{0,0}}{\gamma_{1}}=\frac{1}{p_{1}^{2}-r_{2}}\left(-a_{2}+c_{2} p_{0}+d_{1}\left(3 p_{0} p_{1}+2 r_{1}\right)+d_{0}\left(p_{1}^{2}+2 r_{2}\right)\right. \\
\left.+p_{1}\left(p_{1} d_{0}+p_{0} d_{1}+c_{1}\right)+\frac{\Theta_{0,1}}{\gamma_{1}}\left(r_{1}-2 p_{0} p_{1}\right)\right) .
\end{gathered}
$$

Here, $p_{1}, p_{0}, r_{2}, r_{1}$ are the coefficients of $p(x)$ and $r(x)$ defined in (4), and $q$ is defined by ( 7$)$.

Proof: First, let us we deduce (28)-(29).

Evaluating (22) at $x_{0}:=-p_{0} / p_{1}$ we get, as $\mathbb{M}(x)=p(x)$ (which follows from (3) and (8)),

$$
-A\left(x_{0}\right)-2 r\left(x_{0}\right)\left(\pi_{n+1}+\pi_{n}\right)\left(x_{0}\right)+\gamma_{n+2} \frac{\Theta_{n+1}}{\gamma_{n+2}}\left(x_{0}\right)=\gamma_{n+1} \frac{\Theta_{n-1}}{\gamma_{n}}\left(x_{0}\right) .
$$

Thus, we write

$$
\gamma_{n+2} T_{n+1}=\gamma_{n+1} T_{n-1}+\zeta_{n}
$$

with $T_{n+1}=\Theta_{n+1}\left(x_{0}\right) / \gamma_{n+2}, \zeta_{n}=A\left(x_{0}\right)+2 r\left(x_{0}\right)\left(\pi_{n+1}+\pi_{n}\right)\left(x_{0}\right)$. By multiplying (41) by $T_{n}$ we get

$$
\gamma_{n+2} T_{n} T_{n+1}=\gamma_{n+1} T_{n-1} T_{n}+\zeta_{n} T_{n}, \quad n \geq 0 .
$$


Iterating yields

$$
\gamma_{n+2} T_{n} T_{n+1}=\gamma_{1} T_{-1} T_{0}+\sum_{k=0}^{n} \zeta_{k} T_{k}, \quad n \geq 0,
$$

thus we obtain (28), where we used $T_{-1}=D\left(x_{0}\right)$ (see (24)).

To obtain $\gamma_{1}$ we proceed in two steps: first, by collecting the coefficient of $x$ in (24) and using (25), we get

$$
\Theta_{0,1}=a_{1}+c_{1} p_{0}+c_{0} p_{1}+d_{0}\left(2 p_{0} p_{1}-r_{1}\right)+d_{1}\left(p_{0}^{2}-r_{0}\right) .
$$

Next we have (39),

$$
\frac{\Theta_{0,1}}{\gamma_{1}}=\frac{-a_{3}+c_{2} p_{1}+2 d_{1}\left(p_{1}^{2}+r_{2}\right)}{p_{1}^{2}-r_{2}} .
$$

Combining the two equations above we get (29).

Now, let us we deduce the quantities $\Theta_{n, j} / \gamma_{n+1}$ and $\pi_{n, j}, j=0,1$.

To deduce (30) we start by taking $\beta_{n}=0$ as well as $\mathbb{M}(x)=p(x)$ in (21), and collect the coefficients of $x^{2}$, thus getting

$$
\ell_{n+1,2}=-\ell_{n, 2}-p_{1} \frac{\Theta_{n, 1}}{\gamma_{n+1}} .
$$

Also, starting with the definition of $A_{n}$ in (19), and using a similar procedure as in [5, Lemma 1], we obtain $\Theta_{n+1,1} / \gamma_{n+2}$ as a linear combination of $\ell_{n, 2}$ and $\Theta_{n, 1} / \gamma_{n+1}$,

$$
\frac{\Theta_{n+1,1}}{\gamma_{n+2}}=\frac{-4 p_{1}}{r_{2}-p_{1}^{2}} \ell_{n, 2}+\left(1-\frac{2\left(r_{2}+p_{1}^{2}\right)}{r_{2}-p_{1}^{2}}\right) \frac{\Theta_{n, 1}}{\gamma_{n+1}} .
$$

Then we write (42) and (43) in the matrix form,

$$
\left[\begin{array}{c}
\ell_{n+1,2} \\
\Theta_{n+1,1} / \gamma_{n+2}
\end{array}\right]=\mathcal{X}\left[\begin{array}{c}
\ell_{n, 2} \\
\Theta_{n, 1} / \gamma_{n+1}
\end{array}\right], \quad \mathcal{X}=\left[\begin{array}{cc}
-1 & -p_{1} \\
\frac{-4 p_{1}}{r_{2}-p_{1}^{2}} & 1-\frac{2\left(r_{2}+p_{1}^{2}\right)}{r_{2}-p_{1}^{2}}
\end{array}\right] .
$$

Iterating (44) yields, for all $n \geq 0$,

$$
\begin{aligned}
\ell_{n+1,2} & =\left(\mathcal{X}^{n+1}\right)_{(1,1)} \ell_{0,2}+\left(\mathcal{X}^{n+1}\right)_{(1,2)} \Theta_{0,1} / \gamma_{1}, \\
\Theta_{n+1,1} / \gamma_{n+2} & =\left(\mathcal{X}^{n+1}\right)_{(2,1)} \ell_{0,2}+\left(\mathcal{X}^{n+1}\right)_{(2,2)} \Theta_{0,1} / \gamma_{1} .
\end{aligned}
$$

Here, $\left(\mathcal{X}^{n+1}\right)_{(i, j)}$ denotes the element on the position $(i, j)$ of $\mathcal{X}^{n+1}$. 
The diagonalization of $\mathcal{X}$ proceeds as in [5, Lemma 1]. Indeed, the set of the eigenvalues of $\mathcal{X}$ is given by $\sigma(\mathcal{X})=\left\{q, q^{-1}\right\}$ with $q$ defined in (7). Therefore, we get $\mathcal{X}=\mathcal{V} \mathcal{D} \mathcal{V}^{-1}$, with $\mathcal{V}, \mathcal{D}$ given by

$$
\mathcal{V}=\left[\begin{array}{cc}
\frac{-p_{1}}{1+q} & \frac{-p_{1}}{1+q^{-1}} \\
1 & 1
\end{array}\right], \quad \mathcal{D}=\left[\begin{array}{cc}
q & 0 \\
0 & q^{-1}
\end{array}\right]
$$

Thus, $\mathcal{X}^{n+1}=\mathcal{V} \mathcal{D}^{n+1} \mathcal{V}^{-1}$. As a consequence, from (46) we obtain (30). To get $\ell_{0,2}$ and $\pi_{0,1}$ we use (25) and (23), respectively. The quantity $\Theta_{0,1} / \gamma_{1}$ follows from equating the coefficients of $x^{3}$ in (18) with $n=1$ combined with (21) with $n=0$.

Equation (31) follows from the definition of $\pi_{n}$ (cf. (20)) combined with (30).

Equation (32) follows from (20).

In order to deduce $\pi_{n, 0}$ we start by obtaining some formulae involving $\ell_{n, 1}$. By equating the coefficients of $x^{n+2}$ in (17) we get

$$
\ell_{n, 1}=\frac{c_{1}}{2}+\frac{1}{m_{n+1, n+1}}\left(a_{n+1,3}\left(d_{n+1, n-1}-d_{n+1, n} \frac{m_{n+1, n}}{m_{n+1, n+1}}\right)+a_{n+1,2} d_{n+1, n}\right),
$$

where the $m_{n+1, k}$ and $d_{n+1, k}$ are given by (11). As $a_{n+1,3}=a_{3}+2 r_{2} \pi_{n, 1}, a_{n+1,2}=$ $a_{2}+2 r_{2} \pi_{n, 0}+2 r_{1} \pi_{n, 1}$, we get

$$
\ell_{n, 1}=f_{n}+f_{n, 1} \pi_{n, 1}+f_{n, 0} \pi_{n, 0},
$$

with $f_{n, 0}, f_{n, 1}, f_{n}$ given by (36)-(38). Now, to obtain $\pi_{n, 0}$, we begin by collecting the coefficient of $x$ in (21), hence,

$$
\ell_{n+1,1}+\ell_{n, 1}+p_{1} \frac{\Theta_{n, 0}}{\gamma_{n+1}}+p_{0} \frac{\Theta_{n, 1}}{\gamma_{n+1}}=0 .
$$

Using (47) in (48) as well as $2\left(\pi_{n, 0}-\pi_{n+1,0}\right)=\Theta_{n, 0} / \gamma_{n+1}$ we get, after basic computations,

$$
\pi_{n+1,0}=s_{n} \pi_{n, 0}+t_{n}, \quad n \geq 0,
$$

with $s_{n}, t_{n}$ given by (34)-(35). Thus, we obtain (33).

To obtain $\pi_{0,0}$ we use (23), and to obtain $\Theta_{0,0} / \gamma_{1}$ we take the coefficient of $x^{2}$ in (18) with $n=1$ combined with (21) with $n=0$. 


\section{References}

[1] R. Askey, J. Wilson, Some basic hypergeometric orthogonal polynomials that generalize Jacobi polynomials, Memoirs AMS vol. 54 n. 319, AMS, Providence, 1985.

[2] S. Belmehdi, On semi-classical linear functionals of class $s=1$. Classification and integral representations, Indag. Math. 3, 253-275 (1992).

[3] A. Branquinho and M.N. Rebocho, Characterization theorem for Laguerre-Hahn orthogonal polynomials on non-uniform lattices, J. Math. Anal. Appl. 427, 185-201 (2015).

[4] D. Dominici and F. Marcellán, Discrete semiclassical orthogonal polynomials of class one, Pacific J. Math. 268, 389-411 (2014).

[5] G. Filipuk and M.N. Rebocho, Orthogonal polynomials on systems of non-uniform lattices from compatibility conditions, J. Math. Anal. Appl. 456, 1380-1396 (2017).

[6] M. Foupouagnigni, M. Kenfack Nangho, and S. Mboutngam, Characterization theorem for classical orthogonal polynomials on non-uniform lattices: the functional approach, Integral Transforms Spec. Funct. 22, 739-759 (2011).

[7] R. Koekoek, P.A. Lesky, and R.F. Swarttouw, Hypergeometric Orthogonal Polynomials and Their $q$-Analogues. Springer, Berlin (2010).

[8] S. Mboutngama, M. Foupouagnigni, and P. Njionou Sadjang, On the modifications of semiclassical orthogonal polynomials on nonuniform lattices, J. Math. Anal. Appl. 445, 819-836 (2017).

[9] A.P. Magnus, Riccati acceleration of the Jacobi continued fractions and Laguerre-Hahn polynomials. In: Werner, H., Bunger, H.T. (eds.) Padé Approximation and its Applications (Proceedings Bad Honnef 1983). Lect. Notes in Math., vol. 1071, pp. 213-230. Springer Verlag, Berlin (1984).

[10] A.P. Magnus, Associated Askey-Wilson polynomials as Laguerre-Hahn orthogonal polynomials. In: Alfaro, M., Dehesa, J.S., Marcellán, F.J., Rubio de Francia, J.L., Vinuesa, J. (eds.) Orthogonal Polynomials and Their Applications (Segovia, 1986). Lecture Notes in Math., vol. 1329, pp. 261-278. Springer, Berlin (1988).

[11] A.P. Magnus, Special nonuniform lattice (snul) orthogonal polynomials on discrete dense sets of points, J. Comput. Appl. Math. 65, 253-265 (1995).

[12] P. Maroni and M. Mejri, The symmetric $D_{\omega}$-semiclassical orthogonal polynomials of class one, Numer. Algorithms 49, 251-282 (2008).

[13] A.F. Nikiforov, S.K. Suslov, and V.B. Uvarov, Classical Orthogonal Polynomials of a Discrete Variable (Springer, Berlin, 1991).

[14] A.F. Nikiforov and V.B. Uvarov, Special Functions of Mathematical Physics: A unified Introduction with Applications, Birkhäuser, Basel, Boston, 1988.

[15] A.F. Nikiforov and S.K. Suslov, Classical Orthogonal Polynomials of a discrete variable on non uniform lattices, Letters Math. Phys. 11 (1986) 27-34.

[16] G. Szegő, Orthogonal Polynomials. Amer. Math. Soc. Colloq. Publ. 23. Amer. Math. Soc. Providence, RI (1975) (Fourth Edition).

[17] W. Van Assche, Discrete Painlevé equations for recurrence coefficients of orthogonal polynomials. In: Elaydi, S., Cushing, J., Lasser, R., Ruffing, A., Papageorgiou, V., Van Assche W. (eds.) Difference Equations, Special Functions and Orthogonal Polynomials, pp. 687-725. World Scientific, Hackensack, NJ (2007).

[18] N.S. Witte, Semi-classical orthogonal polynomial systems on nonuniform lattices, deformations of the Askey table, and analogues of isomonodromy, Nagoya Math. J. 219 (2015) 127-234. 
G. FilipuK

Faculty of Mathematics, Informatics and Mechanics, University of Warsaw, Banacha 2, WARSAW, 02-097, POLAND

E-mail address: filipuk@mimuw.edu.pl

M.N. REBOCHO

Departamento de Matemática, Universidade da Beira interior, 6201-001 Covilhã, Portugal; CMUC, Department of Mathematics, University of Coimbra, 3001-501 Coimbra, PORTUGAL

E-mail address: mneves@ubi.pt 